\title{
The immunologic tumor microenvironment in endometrioid endometrial cancer in the morphomolecular context: mutual correlations and prognostic impact depending on molecular alterations
}

\author{
Barbara Willvonseder ${ }^{1} \cdot$ Fabian Stögbauer $^{1} \cdot$ Katja Steiger $^{1,2} \cdot$ Moritz Jesinghaus $^{1,2,3}$. Peer-Hendrik Kuhn ${ }^{1}$. \\ Christine Brambs ${ }^{4}$. Jutta Engel ${ }^{5}$. Holger Bronger ${ }^{4}$. Georg Philipp Schmidt ${ }^{4}$. Bernhard Haller ${ }^{6}$. Wilko Weichert ${ }^{1,2}$. \\ Gisela Keller $^{1} \cdot$ Aurelia Noske $^{1} \cdot$ Nicole Pfarr ${ }^{1} \cdot$ Melanie Boxberg ${ }^{1,2}$
}

Received: 16 May 2020 / Accepted: 26 November 2020 / Published online: 19 December 2020

(c) The Author(s) 2020

\begin{abstract}
Objective POLE-mutant, microsatellite-instable (MSI), p53-mutant and non-specific molecular profile (NSMP) are TCGAdefined molecular subgroups of endometrial cancer (EC). Hypothesizing that morphology and tumor immunology might differ depending on molecular background concerning composition and prognostic impact, we aimed to comprehensively interconnect morphologic, immunologic and molecular data.

Methods TCGA-defined molecular groups were determined by immunohistochemistry and sequencing in $n=142$ endometrioid EC. WHO-defined histopathological grading was performed. The immunologic microenvironment (iTME) was characterised by the quantification of intraepithelial and stromal populations of tumor-infiltrating lymphocytes (TIL: overall T-cells; T-Killer cells; regulatory T-cells (Treg)). Immunologic parameters were correlated with WHO-grading, TCGAsubgroups and prognosis.

Results High density TIL were significantly more frequent in high-grade (G3) compared to low-grade (G1/2) EC in the whole cohort and in the subgroup of POLE-wildtype-/microsatellite-stable-EC. MSI was associated with high-level TILinfiltration when taking into account the type of mismatch repair defect (MLH1/PMS2; MSH2/MSH6). Prognostic impact of biomarkers depended on molecular subgroups: In p53-mutant EC, Treg were independently prognostic, in NSMP, the unique independently prognostic biomarker was WHO-grading.

Conclusions EC morphology and immunology differ depending on genetics. Our study delineated two molecularly distinct subgroups of immunogenic EC characterized by high-density TIL-infiltration: MSI EC and high-grade POLE-wildtype/ microsatellite-stable-EC. Prognostic impact of TIL-populations relied on TCGA-subgroups indicating specific roles for TIL depending on molecular background. In NSMP, histopathological grading was the only prognostic biomarker demonstrating the relevance of WHO-grading in an era of molecular subtyping.
\end{abstract}

Keyword Endometrioid endometrial cancer · Molecular subgroups · Immunologic microenvironment · Prognostic impact

Barbara Willvonseder and Fabian Stögbauer authors contributed equally.

Supplementary Information The online version contains supplementary material available at https://doi.org/10.1007/s0026 2-020-02813-3.

Melanie Boxberg

Melanie.Boxberg@tum.de

Extended author information available on the last page of the article

\section{Introduction}

Endometrial cancer (EC) is the most common gynaecologic malignancy with an estimated number of over 60.000 newly diagnosed cases in the United States accounting for 12.000 deaths in 2019. Incidence and mortality have been rising over the last years [1].

The World Health Organisation (WHO) Classification of Tumours of the Female Reproductive Organs [2] classifies EC into histologic subtypes (e.g. endometrioid; serous; clear 
cell) and defines a histopathologic grading of the endometrioid subtype into G1, G2 (low-grade) and G3 (high-grade; reviewed in [3, 4]) based on the morphologic features glandular vs. solid growth. This classical view has been complemented by insights into molecular genetics. "The Cancer Genome Atlas" (TCGA) published a comprehensive molecular characterisation based on exome sequencing of EC resulting in a classification into four genetically defined subgroups [5]: (1) Polymerase- $\mathcal{E}$ (POLE) ultramutated cases, (2) Microsatellite instable (MSI) cases with defective mismatch repair (MMR) due to the loss of function of MMR proteins MLH1/PMS2 or MSH2/MSH6, (3) Copy number high cases ("serous-like"), characterized by frequent mutations in TP53, extensive copy number variation, low mutational rate and (4) Copy number low microsatellite stable (MSS) cases with no defining molecular alteration (Nonspecific molecular profile; NSMP). Subsequently the TCGA classification was reliably reproduced by two classifiers which are available for routine pathological analysis referred to as ProMisE/Vancouver and PORTEC/Leiden based on the following analysis: POLE mutational analysis by polymerase chain reaction (PCR) followed by mismatch repair protein immunohistochemistry (MMR) and p53 immunohistochemistry [3, 6-8].

The clinical outcome of EC patients varies significantly, suggesting a biological diversity of EC that is not fully reflected in the current models [9]. It is well-recognized that POLE-mutant cases carry an extremely favourable prognosis whereas copy number high cases show an unfavourable outcome necessitating aggressive treatment (reviewed in $[3$, 10]). However, prognostic biomarkers for MSI and NSMP cases remain to be elucidated as these patients show varying clinical courses with an overall intermediate prognosis [5, 10]. Therefore, treatment stratification to avoid under- and overtreatment of patients is difficult, especially in EC with MSI or NSMP. Furthermore, it is unclear if WHO-grading can provide additional information for treatment decisions as the prognostic value of WHO grading in the context of the novel molecular data is not yet elucidated.

Not only knowledge on genetics but as well on tumor immunology has progressed. Comprehensive studies in various tumor entities revealed that a high mutational load with subsequent high numbers of immunogenic neoantigens leads to a strong anti-tumoral cytotoxic T-cell response [11, 12]. In general, based on the presence or absence of T-cells, characterized by expression of CD3 (reviewed in [13]), the immunologic tumor microenvironment (iTME) is classified as "T-cell inflamed" versus "non T-cell inflamed" $[14,15]$. Several subsets of T-cells play-partially antagonising-roles in the immunologic host response: $\mathrm{CD} 8+$ tumor-infiltrating lymphocytes (TIL; T-Killer cells) are crucial for a potent cytotoxic antitumor response [14, 15]. Regulatory T-cells (Treg), specifically characterized by the expression of the transcription factor FoxP3 [16], are involved in the tumorhost interaction by suppression of the immune responses $[4,17]$. In EC, the iTME is-in accordance with the above described mechanisms - composed of several populations of immune cells. Increased numbers of CD3 + TIL have been associated with a favourable [18], FoxP3 + Tregs with a poor outcome [19]. However, studies comprehensively incorporating morphologic, molecular and immunologic data are still rare [12, 20-23] with only few studies comprising comprehensive data on TCGA-defined molecular subgroups.

The overriding hypothesis of our study was, that the role of EC morphology represented by WHO grading as well as influence and infiltration patterns of various TIL populations might differ depending on molecular background (POLE/ P53 mutational status; Microsatellite status). Morphology and iTME might therewith carry differing prognostic impact depending on TCGA grouping of EC and might have the potential to be prognostic biomarkers for specific molecular defined EC subgroups (e.g. MSI and NSMP EC). Targeting these hypotheses, we analysed the immune contexture in a morphologically and molecular well-characterized homogeneous cohort of primary, untreated endometrioid EC. We correlated immunologic with morphologic data and TCGAsubgroups and analysed the prognostic impact of morphologic and immunologic biomarkers in the whole patient cohort and in the context of the TCGA-subgroups. With this study, we present a comprehensive analysis of morphomolecular and immunologic data in endometrioid EC.

\section{Material and Methods}

\section{Patient cohort and clinico-pathological data}

Our cohort included $n=142$ therapy-naïve patients diagnosed with endometrioid EC between 2000 and 2014 who underwent resection of primary tumors at Klinikum Rechts der Isar, Technical University of Munich, Germany. Grading was undertaken according to the current WHO classification of tumors of the female reproductive organs [2]. Staging was performed according to the UICC/FIGO tumor, node and metastasis classification (7th edition; 2011)[24]. Patients received standardized adjuvant treatment and follow-up according to German guidelines [25]. Median follow-up time of patients alive $(88 / 142 ; 62.0 \%)$ was 63.0 months (12.0-191.0 months), median follow-up of deceased patients (54/142; 38.0\%) was 33.0 months (1.0-141.0 months), for the whole patient cohort, median follow-up was 74.0 (5.7) months. Detailed clinico-pathological data are given in Table 1. Approval for the study was obtained from the Ethics Review Committee of the Technical University of Munich (331/17). 
Table 1 Clinicopathological and molecular data

\begin{tabular}{|c|c|c|}
\hline & Number of cases & $\begin{array}{l}\text { Percentage } \\
\text { of cases (\%) }\end{array}$ \\
\hline \multicolumn{3}{|l|}{ Median age (range) } \\
\hline \multicolumn{3}{|l|}{$69.7(36.7-94.0)$} \\
\hline \multicolumn{3}{|l|}{ pT stage } \\
\hline $1 \mathrm{a} / 1 \mathrm{~b}$ & 101 & 71.1 \\
\hline 2 & 24 & 16.9 \\
\hline 3 & 16 & 11.3 \\
\hline N/A & 1 & 0.7 \\
\hline \multicolumn{3}{|l|}{$\mathrm{pN}$ stage } \\
\hline 0 & 132 & 93.0 \\
\hline 1 & 10 & 7.0 \\
\hline \multicolumn{3}{|l|}{ M stage } \\
\hline 0 & 135 & 95.1 \\
\hline 1 & 7 & 4.9 \\
\hline \multicolumn{3}{|l|}{ FIGO Stage } \\
\hline $\mathrm{Ia} / \mathrm{Ib}$ & 100 & 70.4 \\
\hline II & 19 & 13.4 \\
\hline III & 16 & 11.3 \\
\hline IV & 7 & 4.9 \\
\hline \multicolumn{3}{|l|}{ Grading } \\
\hline 1 & 45 & 31.7 \\
\hline 2 & 45 & 31.7 \\
\hline 3 & 52 & 36.6 \\
\hline \multicolumn{3}{|l|}{ p53 status } \\
\hline p53 mutation & 20 & 14.1 \\
\hline p53 wildtype & 122 & 85.9 \\
\hline \multicolumn{3}{|l|}{ Microsatellite status } \\
\hline MSS & 93 & 65.5 \\
\hline MSI (MLH1/PMS2) & 42 & 29.6 \\
\hline MSI (MSH2/MSH6) & 7 & 4.9 \\
\hline \multicolumn{3}{|l|}{ POLE status } \\
\hline POLE mutation & 7 & 4.9 \\
\hline POLE wildtype & 135 & 95.1 \\
\hline \multicolumn{3}{|l|}{ Molecular Classification } \\
\hline POLE-mutant & 7 & 4.9 \\
\hline MSI & 47 & 33.1 \\
\hline P53-mutant & 12 & 8.5 \\
\hline NSMP & 76 & 53.5 \\
\hline
\end{tabular}

\section{Tissue micro array construction}

Formalin-fixed paraffin-embedded tumor samples were assembled into tissue micro arrays (TMA) using a Tissue Microarrayer (Beecher Instruments) with a core size of $0.6 \mathrm{~mm}$. Three cores per case, one from the invasion front and two from tumor core region, were selected from the primary tumors after reviewing the whole tumor slide. Areas were marked taking into account TIL infiltration and were representative for TIL distribution of the whole tumors.

\section{Immunohistochemistry (IHC)}

An automated immunostainer (Ventana Benchmark XT) with an ultraView DAB detection kit (Ventana Medical Systems, Roche) was used for immunohistochemical staining of $2 \mu \mathrm{m}$ sections from the TMA. Primary antibodies against p53 (clone DO-7, dilution 1:200, Dako), MSH2 (clone G219-1129, dilution 1:200, Cell Marque), MSH6 (clone 44, dilution 1:400, BD Transduction Laboratories), PMS2 (clone EPR3974, ready to use, Roche), MLH1 (clone M1, ready to use, Ventana), CD3 (clone MRQ-39, dilution 1:500, Cell Marque), CD8 (clone C8/144B, dilution 1:100, Dako) and FoxP3 (clone 236A/E7, dilution 1:200, Abcam) were applied. Appropriate positive and negative controls were run in parallel.

\section{Scoring of immunohistochemical stainings}

Testing of microsatellite status as well as analysis of p53 status were performed by immunohistochemistry in analogy to ProMisE/Vancouver and PORTEC/Leiden classifiers [3, 6, 8] taking into account all tumor cores. MSI was determined as two markers negative (MSH2/MSH6; MLH1/PMS2). IHC score 0 (complete p53 negativity) or IHC score 2 (strong nuclear p53 positivity in all tumor cells) was used as a surrogate marker for p53-mutation. Intermediate/heterogeneous nuclear staining (score 1) was documented as p53 wild type (Fig. 1, Supplementary Fig. 1).

Staining results of p53, MSH2, MSH6, MLH1 and PMS2 stains on TMA which showed staining results close to respective cut-offs were repeated on whole slides of full tissue blocks to reach a final result for respective stainings.

For analyses of TIL populations (Fig. 1; Supplementary Fig. 1), each tumor core was evaluated separately and the average density across all cores of specific regions (invasive front; tumor center) and whole tumor was calculated. The analysis of the TIL subpopulations was performed in three areas: (1) Intraepithelial TIL: the tumor region of the respective cores showing the highest density of the TIL population was selected on low power magnification $(4 \mathrm{x})$. Within this region, the amount of intraepithelial TIL (CD3i; CD8i; FoxP3i) was scored by counting the absolute number of TIL within 100 tumor cells using high power magnification (40x [26]). (2) Stromal TIL: In analogy to previous TIL-scoring approaches [27] density was evaluated by determination of percentage of the tumor stroma occupied by the respective TIL populations (CD3s; CD8s; FoxP3s). (3) Overall TIL: Density was scored via determination of the percentage of the tumor area (exclusion of necrosis) occupied by the respective TIL population (CD3o; CD8o; FoxP3o) [28].

Absolute values for TIL infiltrates were documented and subsequently assigned to scoring groups as described below. 

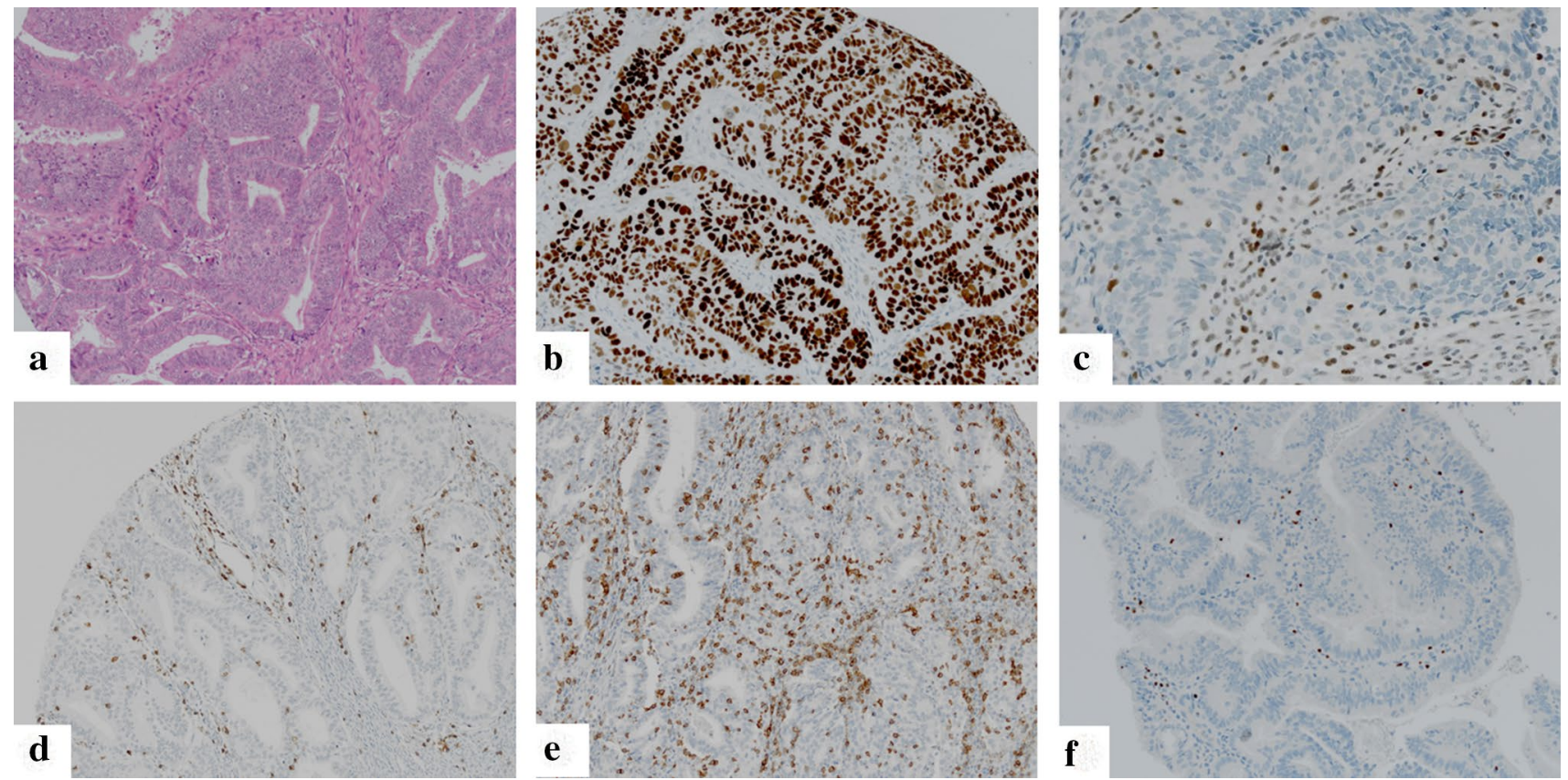

Fig. 1 Representative micrographs of endometrioid endometrial carcinoma: a hematoxylin-eosin staining; b p53 staining showing a p53-mutant carcinoma with immunohistochemical staining score 2; c MLH1 staining showing a carcinoma with loss of MLH1 expres-

\section{Cut-off determination for TIL infiltration}

Using disease-specific survival (DSS) as an endpoint for the determination of the optimal prognostic cut-off values, receiver operating characteristic (ROC) curves were calculated for TIL density and followed by area under the curve (AUC) analysis. Cut-offs for high versus low TIL density were set at values with the highest Youden's index (Supplementary Table 1).

\section{DNA extraction}

After marking of the tumor area and annotation of percentage of vital tumor tissue ( $\geq 50 \%$ tumor cell content) for micro-dissection, DNA was extracted using the Maxwell 16 RSC extraction system (Promega) according to the manufacturer's protocols. DNA concentration was measured fluorometrically using the QuBit 3.0 system (Thermo Fisher Scientific) and DNA quality was determined by a qPCR assay (RNAseP assay, Thermo Fisher Scientific).

\section{POLE (NM.006231) mutational analysis}

All cases were analyzed for POLE- $\varepsilon$-mutations by Sanger sequencing of exons $9,11,13$, and 14, which sion (positive internal control); $\mathbf{d}$ CD3 staining visualizing CD3 + Pan T-cell infiltrate; e; CD8 staining visualizing CD8 + T Killer-cell infiltrate (f) FoxP3 staining with nuclear positivity in regulatory T-cells

were amplified using primers as previously described [29]. Subsequent Sanger sequencing was conducted on a 3130 genetic analyzer (Applied Biosystems) using $5 \mu \mathrm{l}$ amplified DNA/sample and the BigDye Terminator Cycle Sequencing Kit (Applied Biosystems) according to the manufacturers` protocols. Reported files were examined using BioEdit version 7.2.5.

\section{Statistics}

Analyses were performed using SPSS 25 (SPSS Inc.) and $\mathrm{R}$ 3.6.1. The distribution of qualitative data was compared between groups using $\chi^{2}$-test or Fisher's exact test. Survival probabilities were plotted with the cumulative incidence function. Median follow-up was estimated with the Kaplan-Meier estimate for potential follow-up, mean follow-up and 5 year survival by Kaplan Meier estimate. Overall survival (OS) was defined as patients alive at the end of follow-up, disease-specific survival (DSS) as all patients, who did not suffer from disease-related death. Disease-free survival (DFS) included all patients, who did not suffer from disease progression/recurrence during follow-up. Multivariate survival analysis was performed with the Cox Proportional Hazard model. All statistical tests were performed on exploratory two-sided 5\% significance level. 


\section{Results}

\section{Morphologic and molecular characterization of EC}

90/142 (63.4\%) EC were classified as low grade (G1/G2), $52(36.6 \%)$ as high grade (G3). According to the above described classifiers [3, 6-8], the study contained $4.9 \%$ POLE-mutant (detailed list of mutations in Supplementary Table 2), 33.1\% MSI, 8.5\% p53-mutant and 53.5\% NSMP cases (Table 1).

\section{Composition of populations of tumor infiltrating lymphocytes}

Absolute infiltration densities (mean; median; range) of TIL populations are given in Supplementary Table 3. Classification in scoring groups showed the following results ( $\mathrm{o}=$ overall; $\mathrm{i}=$ intraepithelial; $\mathrm{s}=$ stromal): CD $3 \mathrm{i}^{\text {high }}$ $112 / 142$ (78.9\%); CD3s $\mathrm{s}^{\text {high }} 79 / 142$ (55.6\%); CD3o ${ }^{\text {high }}$ $51 / 142$ (35.9\%). CD8i ${ }^{\text {high }} 71 / 142$ (50.0\%); CD8s ${ }^{\text {high }} 103 / 142$ (72.6\%); CD80 ${ }^{\text {high }} 81 / 142$ (57.0\%). FoxP3i ${ }^{\text {high }} 5 / 142(3.5 \%)$; FoxP3s ${ }^{\text {high }} 29 / 142$ (20.4\%); FoxP3o ${ }^{\text {high }} 28 / 142(19.7 \%)$
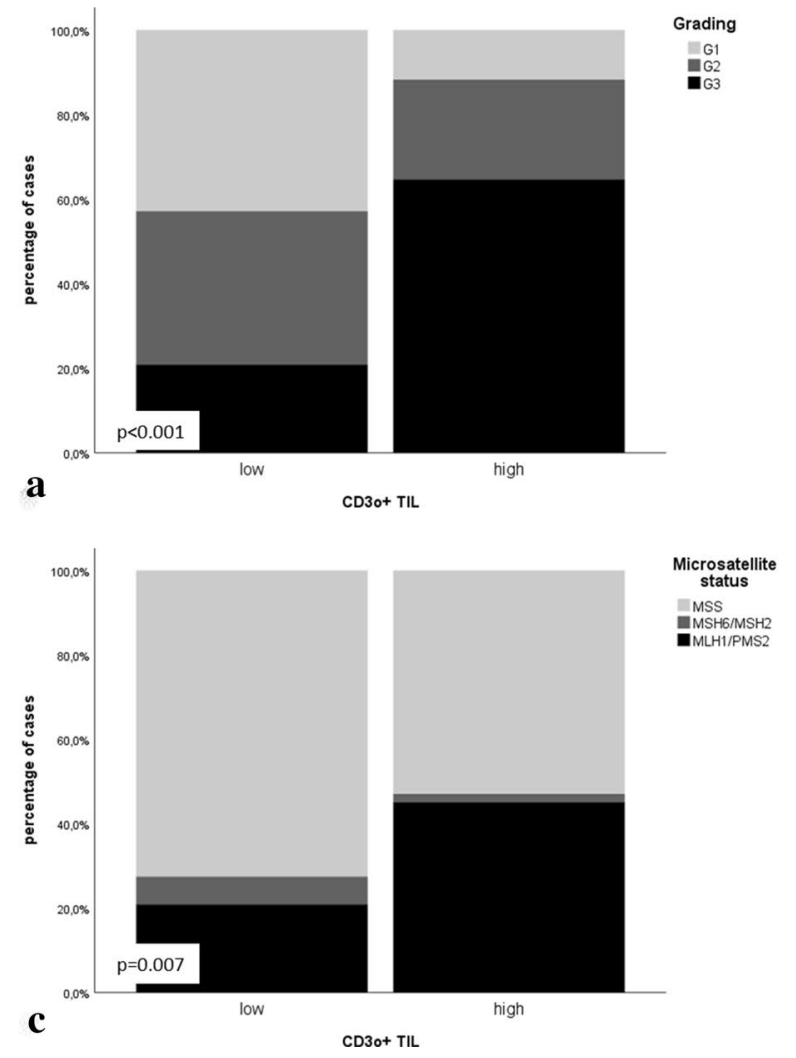

Fig. 2 Association of $\mathrm{CD} 3 \mathrm{o}+$ and $\mathrm{CD} 8 \mathrm{o}+\mathrm{TIL}$ infiltrates (overall infiltration density) with morphomolecular parameters: a CD3o + TIL infiltrate correlated with grading; b CD8o + TIL infiltrate correlated
(Supplementary Table 4; Supplementary Table 5). High density immune cell infiltrate of all analyzed TIL populations was significantly more frequent at the invasive front compared to tumor center $(p<0.01)$.

\section{Correlation of immunologic variables with morphomolecular and clinical data}

Immunologic-morphologic correlation: A high-density Pan T-cell infiltrate $(\mathrm{CD} 3+)$ and T-Killer cell infiltrate (CD8+) was significantly more frequent in high-grade $\mathrm{EC}$ whereas in contrast a high density Treg infiltrate was more frequently observed in low-grade cases ( $p<0.05$; Fig. 2; detailed numbers and $p$ values in Table 2, Supplementary Table 4).

Immunologic-molecular correlations: MSI was associated with $\mathrm{CD} 3+, \mathrm{CD} 8$ + and FoxP3 + T-cell infiltrate when considering the specific mismatch repair defect (MLH1/PMS2 versus MSH2/MSH6): In MLH1/PMS2 deficient EC significantly higher levels of CD3 + ; CD8 + and FoxP3 + TIL infiltrates were observed compared to MSH2/MSH6-deficient and MSS cases (Fig. 2). No significant association of p53 and POLE mutation with immunologic parameters was found, most likely due to only rare mutant cases $(p<0.05$;

b
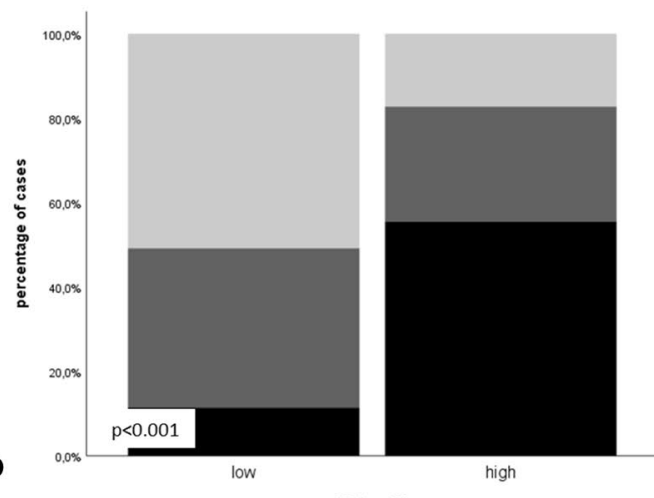

Grading

$\mathrm{G} 1$
$\mathrm{G} 2$
$\mathrm{G} 3$

CD80+ TIL

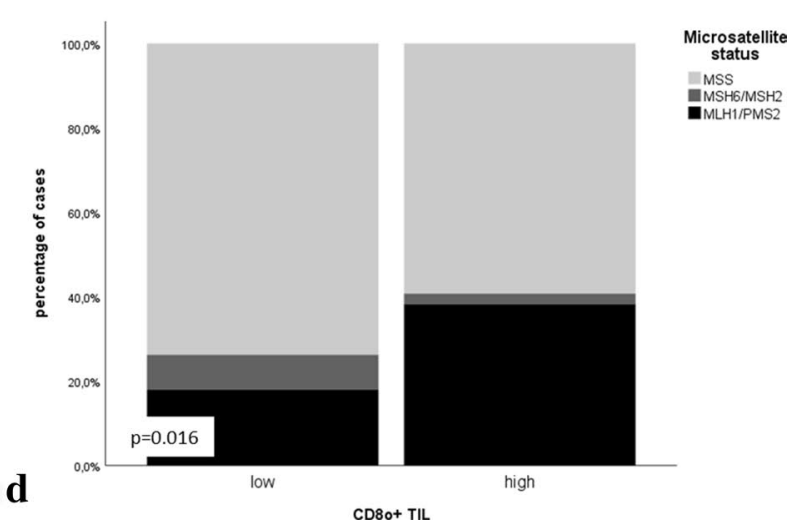

with grading; c CD3o + TIL infiltrate correlated with microsatellite status; d CD8o + TIL infiltrate correlated with microsatellite status 


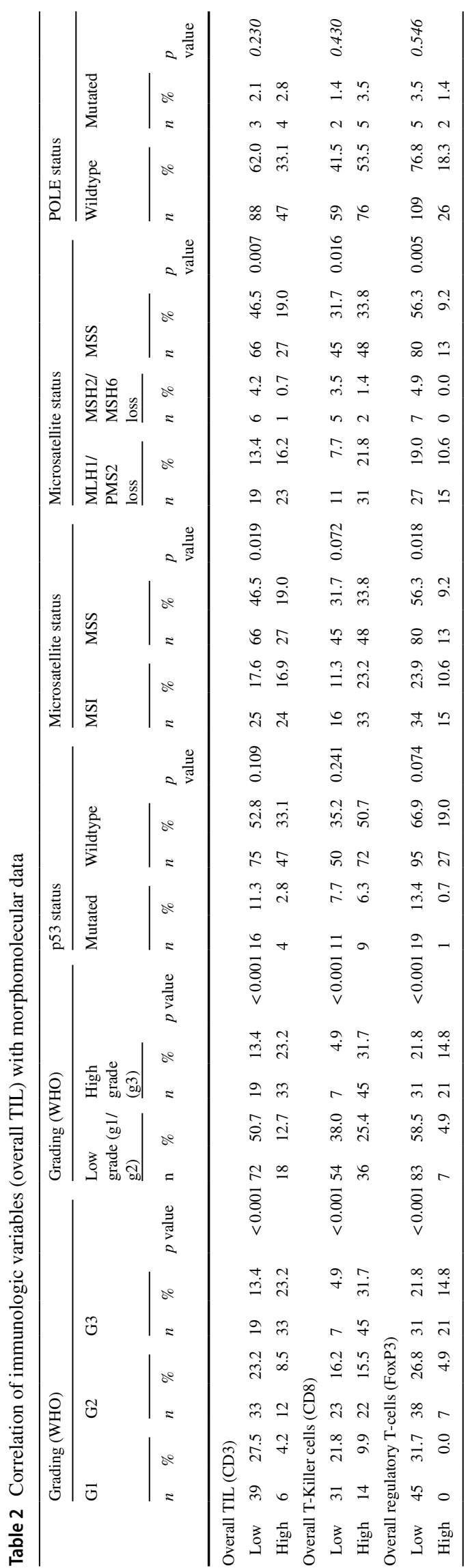

detailed numbers and $p$ values in Table 2, Supplementary Table 4).

Immunologic-clinical correlations: Correlation of immunologic with clinical data revealed only few significant associations. Amongst others, overall Treg (FoxP3o) density was significantly higher in cases with high FIGO stage $(p<0.05$; detailed numbers and $p$ values Supplementary Table 5).

\section{Correlation of immunologic variables with morphomolecular data in the molecular defined subgroup MSS and POLE wildtype EC}

POLE-mutant and MSI EC are known to be highly immunogenic due to their high mutational load. We hypothesized that a further immunogenic subgroup of EC potentially exists among the subgroup of MSS EC without POLE mutation (POLE-wildtype + MSS EC). Therefore, we analyzed associations of immunologic and morphomolecular data in this specific molecular defined subgroup $(n=88)$ : High-density immune cell infiltrate (CD3; CD8; FoxP3) was significantly correlated with high-grade (G3) morphology $(p<0.05$; Supplementary Table 6).

\section{Survival associations of immunologic parameters in the whole patient cohort}

Analysing survival associations in the whole patient cohort, we did not detect any significant correlations (Supplementary Table 7 summarizes OS, DSS and DFS for all immunologic variables).

\section{Survival associations of immunologic parameters depending on TCGA-defined subgroups}

In line with previous literature, molecular classification of EC according to TCGA resulted in four prognostic groups (POLE-ultramutated; MSI; P53-mutant; NSMP) significantly associated with DSS $(p=0.041)$ and DFS $(p=0.014)$ (Supplementary Fig. 2) with POLE-mutant EC carrying the most favourable prognosis and p53-mutant EC showing the worst outcome. These data may prove the robustness of our results.

In p53-mutant EC, stromal Treg were an independent prognostic factor for OS $(p=0.035), \operatorname{DSS}(p=0.032)$ and DFS $(p=0.012)$. Median OS (DSS; DFS) of patients with FoxP3s ${ }^{\text {low }}$ was 83.0 (108.0; 90.0) months compared to 12.0 (12.0; 11.0) months of counterparts (FoxP3s ${ }^{\text {high }}$ ), 5 year survival rates were OS/DSS/DFS: $84.2 \% / 84.2 \% / 73.7 \%$ vs. $0.0 \% / 0.0 \% / 0.0 \%$. Results of cox regression analysis (incorporating age, grading, FIGO stage) are given in Table 3.

In NSMP EC, WHO grading was identified as independent prognostic factor for DSS and DFS whereas immunologic variables did not influence outcome. Patients with 
Table 3 Multivariate statistical analysis for p53-mutant endometrial carcinoma (left side) and endometrial carcinoma with non-specific molecular profile (right side) under inclusion of age, grading, stage and FoxP3 + TIL (in p53-mutant endometrial carcinoma)

\begin{tabular}{|c|c|c|c|c|c|c|c|c|c|}
\hline \multicolumn{5}{|c|}{ Endometrial carcinoma with p53 mutation } & \multicolumn{5}{|c|}{ Endometrial carcinoma with non-specific molecuar profile (NSMP) } \\
\hline & HR (OS) & $\begin{array}{l}\text { Lower CI } \\
(95 \%)\end{array}$ & $\begin{array}{l}\text { Upper CI } \\
(95 \%)\end{array}$ & $p$ value & & HR (DSS) & $\begin{array}{l}\text { Lower CI } \\
(95 \%)\end{array}$ & $\begin{array}{l}\text { Upper CI } \\
(95 \%)\end{array}$ & $p$ value \\
\hline Age & & & & & Age & & & & \\
\hline Per year & 2.755 & 0.369 & 20.560 & 0.323 & Per year & 0.978 & 0.310 & 3.084 & 0.970 \\
\hline Grading & & & & & Grading & & & & \\
\hline 1 & & & & 0.698 & Low & & & & 0.011 \\
\hline 2 & $>25$ & $<0.001$ & $>25$ & & High & 4.329 & 1.404 & 13.349 & \\
\hline 3 & $>25$ & $<0.001$ & $>25$ & & & & & & \\
\hline FIGO Stage & & & & & FIGO Stage & & & & \\
\hline Low (I/II) & 12.477 & 0.682 & $>25$ & 0.089 & Low (I/II) & & & & 0.019 \\
\hline $\begin{array}{l}\text { High (III/ } \\
\text { IV) }\end{array}$ & & & & & $\begin{array}{l}\text { High (III/ } \\
\text { IV) }\end{array}$ & 4.375 & 1.270 & 15.069 & \\
\hline \multicolumn{10}{|l|}{ FoxP3 + TIL } \\
\hline Low & & & & 0.035 & & & & & \\
\hline \multirow[t]{2}{*}{ High } & 49.3 & 1.315 & $>25$ & & & & & & \\
\hline & HR (DSS) & $\begin{array}{c}\text { Lower CI } \\
(95 \%)\end{array}$ & $\begin{array}{c}\text { Upper CI } \\
(95 \%)\end{array}$ & $p$ value & & HR (DFS) & $\begin{array}{c}\text { Lower CI } \\
(95 \%)\end{array}$ & $\begin{array}{c}\text { Upper CI } \\
(95 \%)\end{array}$ & $p$ value \\
\hline Age & & & & & Age & & & & \\
\hline Per year & 1.791 & 0.134 & 23.978 & 0.660 & Per year & 1.572 & 0.561 & 4.401 & 0.390 \\
\hline Grading & & & & & Grading & & & & \\
\hline 1 & & & & 0.726 & Low & & & & 0.042 \\
\hline 2 & $>25$ & $<0.001$ & $>25$ & & High & 2.778 & 1.037 & 7.442 & \\
\hline 3 & $>25$ & $<0.001$ & $>25$ & & & & & & \\
\hline FIGO Stage & & & & & FIGO Stage & & & & \\
\hline Low (I/II) & & & & 0.119 & Low (I/II) & & & & $<0.001$ \\
\hline $\begin{array}{l}\text { High (III/ } \\
\text { IV) }\end{array}$ & 12.377 & 0.518 & $>25$ & & High (III/IV) & 8.527 & 3.144 & 23.123 & \\
\hline \multicolumn{10}{|l|}{ FoxP3 + TIL } \\
\hline Low & & & & 0.032 & & & & & \\
\hline \multirow[t]{2}{*}{ High } & 71.588 & 1.430 & $>25$ & & & & & & \\
\hline & HR (DFS) & $\begin{array}{c}\text { Lower CI } \\
(95 \%)\end{array}$ & $\begin{array}{c}\text { Upper CI } \\
(95 \%)\end{array}$ & $p$ value & & & & & \\
\hline \multicolumn{10}{|l|}{ Age } \\
\hline Per year & 0.477 & 0.050 & 4.506 & 0.518 & & & & & \\
\hline \multicolumn{10}{|l|}{ Grading } \\
\hline 1 & & & & 0.991 & & & & & \\
\hline 2 & $>25$ & $<0.001$ & $>25$ & & & & & & \\
\hline 3 & $>25$ & $<0.001$ & $>25$ & & & & & & \\
\hline \multicolumn{10}{|l|}{ FIGO Stage } \\
\hline Low (I/II) & & & & 0.029 & & & & & \\
\hline $\begin{array}{l}\text { High (III/ } \\
\text { IV) }\end{array}$ & 17.238 & 1.347 & $>25$ & & & & & & \\
\hline \multicolumn{10}{|l|}{ FoxP3 + TIL } \\
\hline Low & & & & 0.012 & & & & & \\
\hline High & 147.060 & 2.976 & $>25$ & & & & & & \\
\hline
\end{tabular}

low-grade EC had a mean DSS/DFS 117.7/127.1 months while mean DSS/DFS of counterparts with high-grade carcinomas was 107.8/112.9 months. 5 year survival rates were as follows: DSS 90.9\%/57.1\%; DFS 81.8\%/57.1\%. Resulting hazard ratios for high-grade EC were 4.2 for DSS $(p=0.11)$ and 2.8 for DFS ( $p=0.042$; Fig. 3; Table 3 ). 

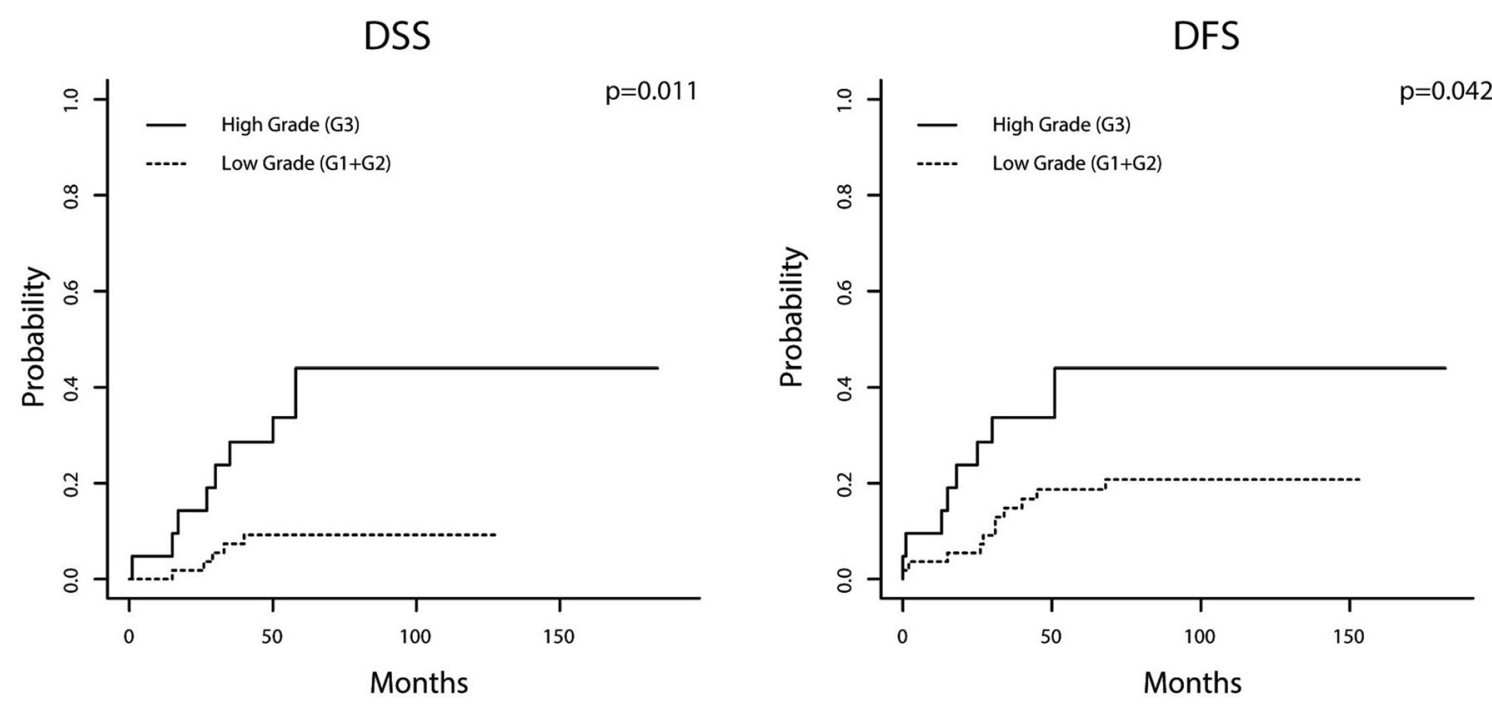

Fig. 3 Cumulative incidence function visualizing disease-specific and disease-free survival depending on grading [carcinomas with non-specific molecular profile $(\mathbf{a}, \mathbf{b})]$

No correlation of immunologic parameters with outcome was observed in POLE-mutant cases.

\section{Discussion}

EC is the malignancy with highest prevalence and incidence in gynaecology. Clinical outcome of patients varies significantly [9] —necessitating prognostic biomarkers to avoid over- or undertreatment. Molecular subgrouping as defined by TCGA into POLE-mutant EC, MSI EC, NSMP EC and p53-mutant EC renders valuable prognostic information with POLE-mutant cases carrying a favourable prognosis whereas P53-mutant EC show an unfavourable outcome necessitating aggressive treatment (reviewed in [3, 10]). However, prognostic biomarkers for MSI and NSMP cases remain to be elucidated as these patients show varying clinical courses with an overall intermediate prognosis [5, 10]. Furthermore, the future prognostic value of morphologic grading described by the WHO classification $[3,4]$ in the molecular context is up to date unclear as e.g. POLE-mutant EC show a high-grade (G3) morphology despite comparably benign clinical course $[6,7,10]$. TCGA-defined molecular groups might not only give prognostic information but may, furthermore, influence the composition and prognostic impact of the iTME of EC consisting of various TIL populations.

Aiming to gain data to further elucidate the role and impact of morphology and tumor immunology in the context of the underlying molecular alterations we analysed the immune contexture represented by the intratumoral T-cell infiltrate (Pan T-cells, T-Killer cells, Treg) in a morphologically and molecularly well-characterized homogeneous cohort of primary, untreated endometrioid EC. We correlated immunologic with morphologic data and TCGAdefined molecular subgroups and analysed the prognostic impact of morphologic and immunologic biomarkers in the whole patient cohort and in the context of TCGA-subgroups.

Confirming previous studies, our data show that subgroups of EC contain a T-cell inflamed immunogenic iTME represented by a dense $\mathrm{T}$-cell infiltration [18, 20-22]. In accordance with our hypothesis outlined in the introduction, these immunogenic subgroups were characterized by distinct morphologic and molecular features:

We observed an immunogenic subgroup characterized by high-grade (G3) morphology: A high-density TIL infiltrate was significantly correlated with WHO-defined high-grade (G3) EC - a finding, which was observed as well in a previous analysis by $\mathrm{Li}$ et al. [30]. The correlation might, at first glance, be explained by a high number of high-grade morphology cases with MSI or POLE-mutation, both of which are known to show high-grade morphology and to be densely infiltrated by immune cells [17, 20, 22, 30-32]. But the correlation was found to be significant in the subgroup of MSS EC without POLE mutation (MSS/POLE wildtype EC), demonstrating, that an immunogenic subgroup of EC without MSI/POLE mutation is characterized by high-grade (G3) morphology.

Furthermore, MSI was confirmed to be a highly immunogenic subtype of EC: MSI EC were significantly associated with a high density TIL infiltrate. In previous studies these finding was linked to a high mutational load in MSI cancers [12, 20, 22, 23, 30, 31]. Interestingly, in our study, the correlation of MSI with high immunogenicity was only significant when the underlying type of MMR defect was included into 
calculation with MLH1/PMS2 defective cases being highly immunogenic in contrast to MSH2/MSH6 defective and MSS EC. This may give a hint that either type of MMR defect or sporadic versus hereditary origin of MSI might imply a very distinct iTME [33].

Interestingly, the inflammatory infiltrate showed heterogeneity considering their intratumoral distribution: the high density T-cell infiltration showed significantly higher levels within the invasive front.

Data concerning prognostic impact of immunologic biomarkers in EC in the context of TCGA-subgroups is up to date rare with previous studies including partially small cohorts with mixed EC subtypes and unclear genetic background $[18,19,23,30]$. In the whole-molecularly highly heterogeneous-patient cohort, none of the analysed variables showed a prognostic impact. In contrast, when considering the molecular background, we found striking differences in the impact of certain T-cell populations on patient prognosis: In p53-mutant cases, Tregs showed a major prognostic impact. A high density Treg infiltrate was independently correlated with a poor patient prognosis. In NSMP EC, immunologic biomarkers revealed no prognostic impact-the only independent biomarker was histopathological WHO-based grading with high-grade EC carrying a significantly worse prognosis.

In accordance with our results, Yamagami et al. found Tregs to be a negative prognostic biomarker [19]. Previous results concerning further TIL populations were inconclusive: Yamashita et al. found an impact of CD8 + TIL on DFS [23] but only used univariate analysis. Cermakova identified CD3 + T-cell infiltrate to be prognostic but did not further subtype the TIL subpopulation [18]. These differing results may at least in part be explained by the fact that none of the previous studies analysed the prognostic impact of immunologic parameters in the context of TCGA subgroups.

The prognostic impact of Treg may be explained by the functional role of these lymphocyte populations in cancer immunity. Tregs suppress immune responses by suppression of activation, proliferation and effector functions of numerous cell types including T-Killer cells and are, therefore, involved in metastasis and progression [17]. In line with that, a high density Treg infiltrate may indicate a poor survival due to suppression of anti-cancer immune responses.

Taking together the results of our study the presented comprehensive analysis of morphology and iTME in the context of the TCGA-defined subgroups delineates major immunogenic EC subgroups: MSI EC, especially those with MLH1/PMS2 defect, and high-grade (G3) POLE-wildtype/ MSS EC. We, furthermore, identify prognostic TIL subpopulations (Treg), which vary depending on TCGA subgroups indicating specific roles for TIL populations depending on molecular background. In NSMP EC, WHO grading was the only independent prognostic biomarker demonstrating the potential future relevance of WHO-grading in an era of molecular subtyping.

Our study clearly has some limitations. Potentially due to the low absolute number of cases with POLE mutation (which is nevertheless in accordance with the literature [34, 35]), we did not detect associations of POLE-mutant EC (which had been demonstrated in multiple previous studies) with T-cell infiltration. Furthermore, our study has a retrospective design necessitating prospective studies to confirm the findings.

Acknowledgement The authors thank the members of the Munich Biobank and the members of the comparative experimental pathology unit for their technical support.

Author contributions BW: Data acquisition, data analysis, methodology, project administration, writing — original draft; FS: Data acquisition, data analysis, methodology, project administration, writingoriginal draft; KS: Data acquisition, writing —original draft; MJ: Data acquisition, writing — original draft; P-HK: Data acquisition, writing original draft; CB: Clinical Data acquisition, writing—original draft; JE: Clinical Data acquisition, writing_original draft; HB: Clinical Data acquisition, writing — original draft; GPS: Clinical Data acquisition, writing —original draft; BH: Statistical Analysis, writing —original draft; WW: Data acquisition; writing_original draft; GK: Data acquisition, methodology, project administration, writing-original draft; AN: Data acquisition, methodology, project administration, writing — original draft; NP: Data acquisition, methodology, writingoriginal draft; MB: Data acquisition, data analysis, methodology, study design, supervision, project administration, writing—original draft.

Funding Open Access funding enabled and organized by Projekt DEAL.. Melanie Boxberg received funding by the Else-Kröner-Fresenius Stiftung.

\section{Compliance with ethical standards}

Conflicts of interest The authors declare that they do not have any conflicts of interest.

Ethics approval Approval for the study was obtained from the Ethics Review Committee of the Technical University of Munich (331/17).

Open Access This article is licensed under a Creative Commons Attribution 4.0 International License, which permits use, sharing, adaptation, distribution and reproduction in any medium or format, as long as you give appropriate credit to the original author(s) and the source, provide a link to the Creative Commons licence, and indicate if changes were made. The images or other third party material in this article are included in the article's Creative Commons licence, unless indicated otherwise in a credit line to the material. If material is not included in the article's Creative Commons licence and your intended use is not permitted by statutory regulation or exceeds the permitted use, you will need to obtain permission directly from the copyright holder. To view a copy of this licence, visit http://creativecommons.org/licenses/by/4.0/. 


\section{References}

1. Siegel RL, Miller KD, Jemal A (2019) Cancer statistics, 2019. CA Cancer J Clin 69:7-34. https://doi.org/10.3322/caac.21551

2. Kurman RJ, Carcangiu ML, Herrington S, Young RH (2014) WHO classification of tumours of female reproductive organs. IARC

3. Soslow RA, Tornos C, Park KJ et al (2019) Endometrial carcinoma diagnosis: use of FIGO grading and genomic subcategories in clinical practice: recommendations of the international society of gynecological pathologists. Int J Gynecol Pathol 38(Suppl 1):S64-S74. https://doi.org/10.1097/PGP.0000000000000518

4. Lakshmi Narendra B, Eshvendar Reddy K, Shantikumar S, Ramakrishna S (2013) Immune system: a double-edged sword in cancer. Inflamm Res 62:823-834. https://doi.org/10.1007/s0001 1-013-0645-9

5. Cancer Genome Atlas Research N, Kandoth C, Schultz N et al (2013) Integrated genomic characterization of endometrial carcinoma. Nature 497:67-73. https://doi.org/10.1038/nature12113

6. Talhouk A, McConechy MK, Leung S et al (2015) A clinically applicable molecular-based classification for endometrial cancers. Br J Cancer 113:299-310. https://doi.org/10.1038/bjc.2015.190

7. Talhouk A, McConechy MK, Leung S et al (2017) Confirmation of ProMisE: a simple, genomics-based clinical classifier for endometrial cancer. Cancer 123:802-813. https://doi.org/10.1002/ cncr.30496

8. Stelloo E, Nout RA, Osse EM et al (2016) Improved risk assessment by integrating molecular and clinicopathological factors in early-stage endometrial cancer-combined analysis of the PORTEC cohorts. Clin Cancer Res 22:4215-4224. https://doi. org/10.1158/1078-0432.CCR-15-2878

9. McAlpine J, Leon-Castillo A, Bosse T (2018) The rise of a novel classification system for endometrial carcinoma; integration of molecular subclasses. J Pathol 244:538-549. https://doi. org/10.1002/path.5034

10. Talhouk A, McAlpine JN (2016) New classification of endometrial cancers: the development and potential applications of genomicbased classification in research and clinical care. Gynecol Oncol Res Pract 3:14. https://doi.org/10.1186/s40661-016-0035-4

11. Chen DS, Mellman I (2017) Elements of cancer immunity and the cancer-immune set point. Nature 541:321-330. https://doi. org/10.1038/nature21349

12. Howitt BE, Shukla SA, Sholl LM et al (2015) Association of polymerase e-mutated and microsatellite-instable endometrial cancers with neoantigen load, number of tumor-infiltrating lymphocytes, and expression of PD-1 and PD-L1. JAMA Oncol 1:1319-1323. https://doi.org/10.1001/jamaoncol.2015.2151

13. Abbas AK, Lichtman AH, Pillai S (2014) Cellular and molecular immunology E-book. Elsevier Health Sciences, Amsterdam

14. Teng MW, Ngiow SF, Ribas A, Smyth MJ (2015) Classifying cancers based on T-cell infiltration and PD-L1. Cancer Res 75:2139_ 2145. https://doi.org/10.1158/0008-5472.CAN-15-0255

15. Spranger $S$ (2016) Mechanisms of tumor escape in the context of the T-cell-inflamed and the non-T-cell-inflamed tumor microenvironment. Int Immunol 28:383-391. https://doi.org/10.1093/intim $\mathrm{m} / \mathrm{dxw} 014$

16. Romagnani S (2014) T cell subpopulations. Chem Immunol Allergy 100:155-164. https://doi.org/10.1159/000358622

17. Liu S, Liu D, Li J, Zhang D, Chen Q (2016) Regulatory T cells in oral squamous cell carcinoma. J Oral Pathol Med: Off Publ Int Assoc Oral Pathol Am Acad Oral Pathol 45:635-639. https://doi. org/10.1111/jop. 12445

18. Cermakova P, Melichar B, Tomsova M, Zoul Z, Kalabova H, Spacek J, Dolezel M (2014) Prognostic significance of CD3+ tumor-infiltrating lymphocytes in patients with endometrial carcinoma. Anticancer Res 34:5555-5561

19. Yamagami W, Susumu N, Tanaka H, Hirasawa A, Banno K, Suzuki N, Tsuda H, Tsukazaki K, Aoki D (2011) Immunofluorescence-detected infiltration of $\mathrm{CD} 4+\mathrm{FOXP} 3+$ regulatory $\mathrm{T}$ cells is relevant to the prognosis of patients with endometrial cancer. Int J Gynecol Cancer 21:1628-1634. https://doi.org/10.1097/ IGC.0b013e31822c271f

20. Asaka S, Yen TT, Wang TL, Shih IM, Gaillard S (2019) T cellinflamed phenotype and increased Foxp3 expression in infiltrating T-cells of mismatch-repair deficient endometrial cancers. Mod Pathol 32:576-584. https://doi.org/10.1038/s41379-018-0172-x

21. Crumley S, Kurnit K, Hudgens C, Fellman B, Tetzlaff MT, Broaddus R (2019) Identification of a subset of microsatellite-stable endometrial carcinoma with high PD-L1 and CD8+ lymphocytes. Mod Pathol 32:396-404. https://doi.org/10.1038/s4137 9-018-0148-x

22. Eggink FA, Van Gool IC, Leary A et al (2017) Immunological profiling of molecularly classified high-risk endometrial cancers identifies POLE-mutant and microsatellite unstable carcinomas as candidates for checkpoint inhibition. Oncoimmunology 6:e1264565. https://doi.org/10.1080/2162402X.2016.1264565

23. Yamashita H, Nakayama K, Ishikawa M et al (2018) Microsatellite instability is a biomarker for immune checkpoint inhibitors in endometrial cancer. Oncotarget 9:5652-5664. https://doi. org/10.18632/oncotarget.23790

24. Sobin LH, Gospodarowicz MK, Wittekind C (2011) TNM classification of malignant tumours. John Wiley \& Sons, USA

25. Emons G (2009) Endometriumkarzinom. Der Gynäkologe 42:799

26. Boxberg M, Leising L, Steiger K et al (2019) Composition and clinical impact of the immunologic tumor microenvironment in oral squamous cell carcinoma. J Immunol 202:278-291. https:// doi.org/10.4049/jimmunol.1800242

27. Fehrenbacher L, Spira A, Ballinger M et al (2016) Atezolizumab versus docetaxel for patients with previously treated non-smallcell lung cancer (POPLAR): a multicentre, open-label, phase 2 randomised controlled trial. Lancet 387:1837-1846. https://doi. org/10.1016/S0140-6736(16)00587-0

28. Rosenberg JE, Hoffman-Censits J, Powles T et al (2016) Atezolizumab in patients with locally advanced and metastatic urothelial carcinoma who have progressed following treatment with platinum-based chemotherapy: a single-arm, multicentre, phase 2 trial. Lancet 387:1909-1920. https://doi.org/10.1016/S0140 -6736(16)00561-4

29. Stenzinger A, Pfarr N, Endris V et al (2014) Mutations in POLE and survival of colorectal cancer patients-link to disease stage and treatment. Cancer Med 3:1527-1538. https://doi.org/10.1002/ cam4.305

30. Li Z, Joehlin-Price AS, Rhoades J, Ayoola-Adeola M, Miller K, Parwani AV, Backes FJ, Felix AS, Suarez AA (2018) Programmed death ligand 1 expression among 700 consecutive endometrial cancers: strong association with mismatch repair protein deficiency. Int J Gynecol Cancer 28:59-68. https://doi.org/10.1097/ IGC.0000000000001120

31. Bregar A, Deshpande A, Grange C et al (2017) Characterization of immune regulatory molecules B7-H4 and PD-L1 in low and high grade endometrial tumors. Gynecol Oncol 145:446-452. https:// doi.org/10.1016/j.ygyno.2017.03.006

32. Pakish JB, Jazaeri AA (2017) Immunotherapy in gynecologic cancers: are we there yet? Curr Treat Options Oncol 18:59. https ://doi.org/10.1007/s11864-017-0504-y

33. Pakish JB, Zhang Q, Chen Z et al (2017) Immune microenvironment in microsatellite-instable endometrial cancers: hereditary or sporadic origin matters. Clin Cancer Res 23:4473-4481

34. An HJ, Kim KI, Kim JY et al (2007) Microsatellite instability in endometrioid type endometrial adenocarcinoma is associated with 
poor prognostic indicators. The Am J Surg Pathol 31:846-853. https://doi.org/10.1097/01.pas.0000213423.30880.ac

35. Goodfellow PJ, Buttin BM, Herzog TJ et al (2003) Prevalence of defective DNA mismatch repair and MSH6 mutation in an unselected series of endometrial cancers. Proc Natl Acad Sci USA 100:5908-5913. https://doi.org/10.1073/pnas.1030231100
Publisher's Note Springer Nature remains neutral with regard to jurisdictional claims in published maps and institutional affiliations.

\section{Affiliations}

\section{Barbara Willvonseder ${ }^{1} \cdot$ Fabian Stögbauer $^{1} \cdot$ Katja Steiger $^{1,2} \cdot$ Moritz Jesinghaus $^{1,2,3} \cdot$ Peer-Hendrik Kuhn ${ }^{1}$.} Christine Brambs ${ }^{4}$. Jutta Engel ${ }^{5}$. Holger Bronger ${ }^{4}$. Georg Philipp Schmidt ${ }^{4}$. Bernhard Haller ${ }^{6}$. Wilko Weichert ${ }^{1,2}$. Gisela Keller ${ }^{1} \cdot$ Aurelia Noske $^{1} \cdot$ Nicole Pfarr $^{1} \cdot$ Melanie Boxberg ${ }^{1,2} \mathbb{C}$

1 Institute of Pathology, Technical University Munich, Trogerstrasse 18, 81675 Munich, Germany

2 German Cancer Consortium (DKTK), Partner Site Munich, Munich, Germany

3 German Cancer Consortium (DKTK), Partner Site Munich, Institute for Translational Cancer Research, Munich, Germany
4 Department of Obstetrics and Gynecology, Technical University of Munich, Munich, Germany

5 Munich Cancer Registry, Institute for Medical Information Processing, Biometry and Epidemiology (IBE), Ludwig-Maximilians-University (LMU), Munich, Germany

6 Institute for Epidemiology and Statistics, Technical University Munich, Munich, Germany 